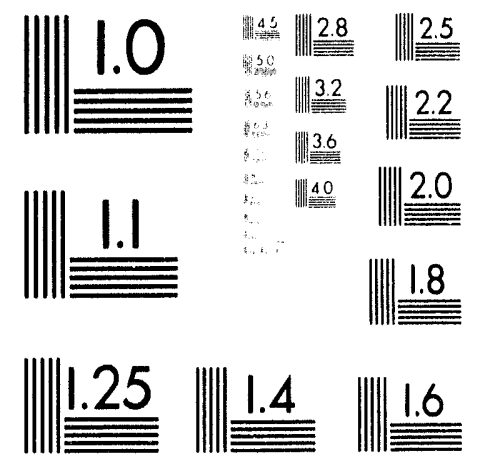



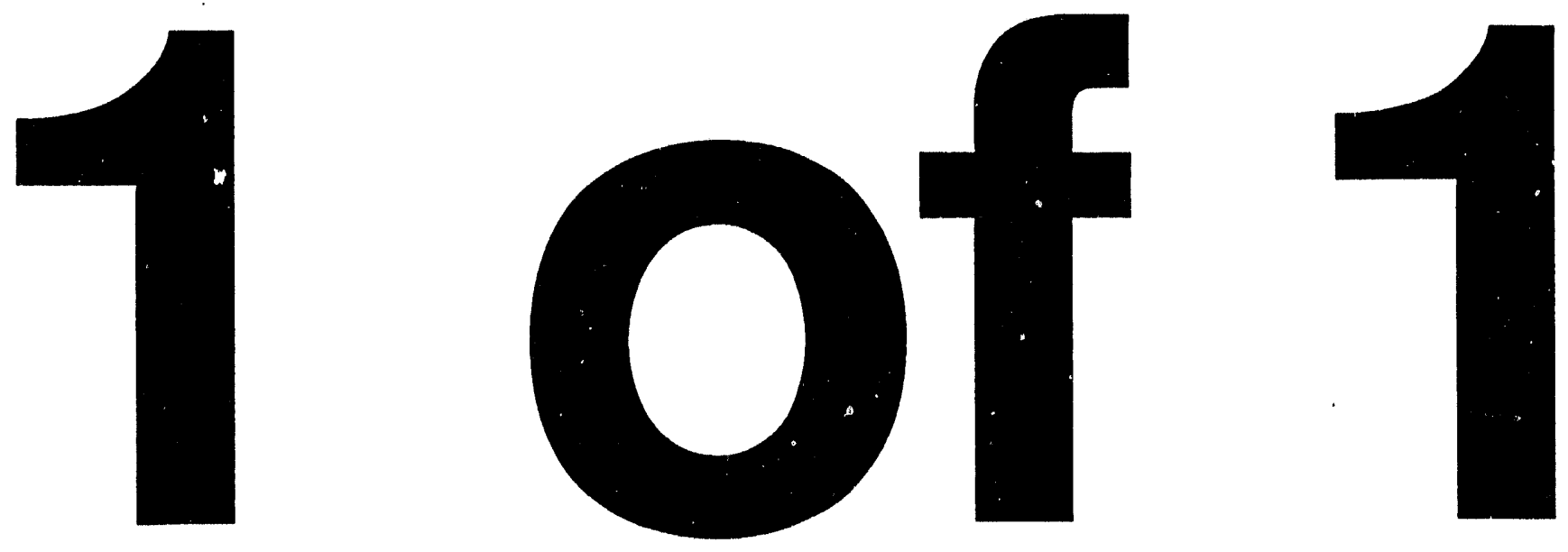
TITLE: Coronal Heating via Nanoflares

Author(S): Giannina Poletto, Osservatorio Astı ofisico di Arcetri Roger Kopp, Los Alamos National Laboratory
SUBMITTED TO: 7th European Meeting on Solar Physics
Topical conference: Advances in Solar Physics
Catania, Italy
May 11-15, 1993

\section{DISCLAIMER}

This report was prepared as an account of work sponsored by an agency of the United States Government. Neither the United States Government nor any agency thureof, nor any of their employees, makes any warranty, express or implied, or assumes any legal liability or responsibility for the accuracy, completeness, or usefulness of any information, apparatus, product, or process disclosed, or represents that its use would not infringe privately owned rights. Reference herein to any specific commercial product, process, or service by trade name, trademark, manufacturer, or otherwise does not necessarily constitute or imply its endorsement, recommendation, or favoring by the United States Government or any agency thereof. The views and opinions of authors expressed herein do not necessarily state or reflect those of the United States Government or any agency thereof.

\section{WEIVED}

\section{OCT $0>1993$ OSTI}

By acceptance of this arlicle the publisher recognizes that the US Government retains a nonexclusive, royalty-lree license to publish or reproduce the published form of this contribution. or to allow others to do so, lor US Government purposes

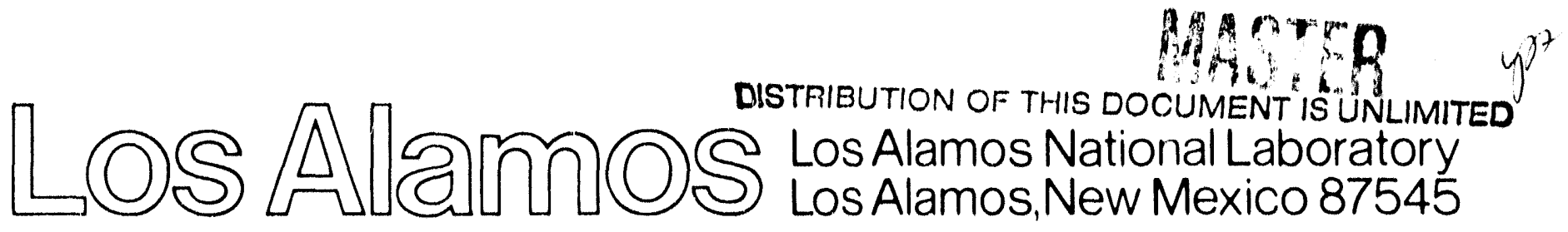




\title{
Coronal Heating via Nanoflares
}

\author{
Giannina Poletto ${ }^{1}$, Roger Kopp ${ }^{2}$ \\ ${ }^{1}$ Osservatorio Astrofisico di Arcetri, Largo Enrico Fermi 5, 50125 Firenze, \\ Italy \\ ${ }^{2}$ Los Alamos National Laboratory, Los Alamos, NM 87545, USA
}

\begin{abstract}
It has been recently proposed that the coronae of single late-type main sequence stars represent the radiative output from a large number of tiny energy release events, the so-called nanoflares. Although this suggestion is attractive and order of magnitude estimates of the physical parameters involved in the process are consistent with available data, nanoflares have not yet been observed and theoretical descriptions of these phenomena are still very crude. In this paper we examine the temporal behavior of a magnetic flux tube subject to the repeated occurrence of energy release events, randomly distributed in time, and we show that an originally empty cool loop may, in fact, reach typical coronal density and temperature values via nanoflare heating. By choosing physical parameters appropriate to solar conditions we also explore the possibilities for observationally detecting nanoflares. Although the Sun is the only star where nanoflares might be observed, present instrumentation appears to be inadequate for this purpose.
\end{abstract}

\section{The Coronal Heating Puzzle}

The coronal heating mechanism has been a subject of debate over the last 40 years and still is not unambiguously identified. Following the acoustic wave heating hypothesis proposed around 1950, alternative processes, in which magnetic fields play a crucial role, were advanced as a result of observations acquired from space-borne experiments. Satellite data revealed that the solar corona consists of a hot gas whose radiative output occurs largely in the previously unaccessible $\mathrm{X}$-ray region and is mainly concentrated in magnetically active areas. Subsequent observations of X-ray emission from other objects showed the presence of hot coronae in middle and lower main sequence stars. All this information was consistent with the hypothesis that the ultimate source of the energy responsible for the formation of stellar coronae resides in the convection zone. However, although the coronal energy requirement is easily met, neither acoustic, slow-mode, nor Alfvén waves seem capable of depositing the right amount of energy in the correct location. The situation had come nearly to a standstill, when new observations suggested a different approach to the problem.

Beginning in the late 70's Skylab, OSO-8, and subsequent missions reported the ubiquitous presence on the Sun of UV and X-ray small-scale, short-duration impulsive events that appeared to be the low-energy analogy of "classical" flares. 
At present this family of mini-events includes a varicty of members - UV jets, UV turbulent events, and hard X-ray evenrs, among others - whose mutual interrelationship is not clear. These data also show that UV microflares, for instance, are much more frequent and widespread than are larger flares: Porter et al. (1984) reported almost continuous activity at the brightest sites of solar active regions over time intervals on the order of hours. More recently, Porter et al. (1992) have also shown that CIV microflares have impulsive counterparts in $3.5-5.5 \mathrm{keV} \mathrm{X}$-ray emission. Hence, microflares are "real" flares, reaching temperatures in excess of that of the ambient corona.

These data raise a question: can this poorly explored population of small flares contribute significantly to the formation of coronae? Can an unobserved swarm of still smaller events, the nanoflares, - with energy releases typically on the order of $10^{-9}$ that of a large solar flare - be responsible for the existence of the solar corona itself? This question addresses not only the solar case: according to Butler et al. (1986), EXOSAT observations of flare stars show evidence for a microflare-heated corona in dMe stars and possibly in all late-type stars. Although neither the solar nor the stellar data are unambiguous, they seem to lead toward the same conclusion.

From a theoretical viewpoint, the formation of the corona as a result of energy release from a multitude of small-scale events has sporadically shown up in the literature, starting from a suggestion of Gold (1964), through Levine (1974), Glencross (1975), Heyvaerts and Priest (1984), up to the recent works of Parker (1983, 1988, 1991). The latter author has pointed out that, whenever field-line footpoints wander randomly around each other (as a consequence of photospheric motions), a large number of localized tangential discontinuities arise. The nanoflares that purportedly build up the corona may originate via magnetic reconnection and dissipation processes localized at these discontinuities. An order of magnitude estimate of the nanoflare energy release, based on typical values of scale-length, footpoint velocity, and magnetic field strength in the solar atmosphere, supports the view of the solar corona as being maintained in a quasi-steady state by an ongoing succession of a great many nanoflares.

However, much remains to be done, both observationally and theoretically. In the solar atmosphere, neither individual nanoflares nor moving magnetic fibrils have been detected. From a theoretical point of view, there are a number of unexplored issues - not even the physics of the reconnection process giving rise to nanoflares has been adequately developed. In the present work, we consider the following problem. Assuming a nanoflare population - whose origin is not discussed here - we investigate whether these events are capable of building up a high-temperature, low-density, quasistatic corona. This question is not a trivial one, as it has still to be demonstrated that a cool empty loop, subject to repeated energy release events, may eventually reach a quasisteady state with the observed coronal properties, via the complex interplay of conduction, radiation, evaporation and gravitational settling. The next section offers a solution to this problem; our conclusions are drawn in Section 3. 


\section{Modeling a Nanoflare-Heated Corona}

The temporal behavior of a magnetic loop subject to an energy release event has been dealt with by a number of authors, who described flaring loops both in the Sun and in stars (see, e.g., Vesecky et al. 1979, Pallavicini et al. 1983, Fisher et al. 1985, MacNeice 1986, Mariska 1987, Reale et al. 1988, Cheng and Pallavicini 1991). Usually detailed hydrodynamical codes are used to integrate numerically the full time-dependent partial differential equations for conservation of mass, momentum, and energy. These models predict the temporal profiles of temperature $(T)$, density $(\rho)$, and velocity $(v)$ as functions of position along the flaring loop. The complexity of the method is justified when, as in the solar case, model predictions can be checked against high-spatial resolution observations. However, for stellar flares as well as for solar and stellar nanoflares, spatial resolution is entirely lacking and a less sophisticated, more flexible method can be adopted. Namely, analytically integrating the conservation equations for mass and energy over the loop semilength $L$, the temporal variations of the spatially averaged loop pressure and density ${ }^{1}$ are given by the following set of total differential equations:

$$
\begin{gathered}
L \frac{d \bar{\rho}}{d t}=(\rho v)_{b} \approx \overline{\rho v} . \\
\frac{L}{\gamma-1} \frac{d \bar{P}}{d t}=\overline{Q_{F}(t)} L+\frac{\gamma}{\gamma-1} \bar{P}_{d}-\left(F_{r}-F_{r 0}\right),
\end{gathered}
$$

where $\bar{P}$ is the loop pressure; $\gamma$ is the specific heat ratio $(=5 / 3) ; F_{r}$ and $F_{r 0}$ are, respectively, the flare and pre-flare radiative loss rates ${ }^{2} ; \overline{Q_{F}(t)}$ is the transient nanoflare energy input; $v_{b}$ is the net plasma velocity at the base of the flux tube and the downflow velocity $\bar{v}_{d}$ is expressed in terms of the free-fall speed $v_{f f}$ for a semicircular loop of height $h=2 L / \pi$ :

$$
\bar{v}_{d}=-\left(1-\bar{\rho}_{0} / \bar{\rho}\right) v_{f f},
$$

where $v_{f f} \equiv \sqrt{2 g h}$. We refer the reader to Kopp and Poletto (1993) for a detailed description of the technique and assumptions which lead to Eqs. 1-3.

In order to predict the behavior of a particular nanoflare-heated loop over an extended time interval, we need to know how many nanoflares per unit time occur on the loop. This is given by the nanoflare energy distribution and by the time-averaged power input to the corona. The latter is known to be $\approx 10^{6} \mathrm{ergs} \mathrm{cm}^{-2} \mathrm{~s}^{-1}$, for the quiet solar corona (Withbroe and Noyes 1977). The nanoflare distribution function, on the other hand, is poorly defined, even for the Sun (Hudson 1991). We know that the number $N$ of flares with total radiated energy $W$ follows approximately a power-law distribution

$$
d N / d W=A W^{-\alpha}(\text { ergs } s)^{-1},
$$

where $\mathcal{A}$ is a constant and $\alpha \approx 1.8$. However, the value of $\alpha$ is well-defined only for the population of "classical" flares; it becomes uncertain when microflares are

${ }^{1}$ From here on an overbar is used for all quantities averaged over the loop semilength

2 These quantities are expressed in terms of an equivalent energy flux at the footpoint 
considered and is totally unkown for nanoflares. A knowledge of its precise value is critical: only distributions with $\alpha>2$ support the hypothesis of a nanoflare-heated corona. Distributions with $\alpha<2$ are dominated by highly energetic flares, which are too few in number to make a significant contribution to the heating of the corona. Recent work by Porter et al. (1992) gives a tentative value $\alpha \approx 2.2$ in the microflare range. In the following we adopt this value also for the exponent of the power-law nanoflare energy distribution and we assume a value $10^{23}$ ergs for its low-energy cutoff.

If we make the simplifying hypothesis that the solar corona is entirely filled with loops with a semilength $L=2000 \mathrm{~km}$ and aspect ratio $(R / L)=0.1$, we can estimate the rate of nanoflare occurrence per loop corresponding to the coronal energy requirement. It turns out that, on the average, a given loop experiences one nanoflare every 239 seconds. Figure 1 shows the behavior predicted by our model for an initially cool $\left(T=10^{5} \mathrm{~K}\right)$ and nearly empty $\left(P \leq 0.001\right.$ dynes $\left.\mathrm{cm}^{-2}\right)$ loop, for which, over the time interval of $2388 s$ covered by the Figure, 10 nanoflares occur randomly distributed in time and energy, although following the aforementioned power-law energy distribution function. Figure 1 shows that temperature and pressure quickly reach values typical of the coronal plasma and support our contention that nanoflares are a viable means to heat the solar corona.

\section{Discussion and Conclusions}

The simulations shown in Section 2 illustrate how a quasi-static corona may be created via the nanoflare heating mechanism. However, considering the capabilities of present-day solar instrumentation, one has been unable, so far, to image a single loop, if its size is that assumed in our modeling. An instrument with a spatial resolution of 3 ", for instance, will include $\approx 6$ such loops within a single pixel. Obviously, the brightness fluctuation from such a loop ensemble is much smoother than would be predicted from the spiky behavior of $T$ and $P$ shown in Figure 1, and an observational test of the theory becomes difficult. Besides, Figure 1 gives an optimistic view of the real situation: longer loops or power-law distributions with higher values of $\alpha$ provide less favorable scenarios, as far as detectability is concerned; we refer the reader to Kopp and Poletto (1993) for a thorough discussion of this point. The situation is, of course, even more discouraging for stars other than the Sun.

Nevertheless, nanoflare heating offers an appealing alternative to more traditional approaches to the coronal heating problem, and it continues to be supported by theoretical and indirect observational evidence. Still, a number of steps should be taken to put this hypothesis on a firmer ground. We mentioned the need for higher spatial resolution observations both at photospheric levels - where fibril motions could be detected - and at coronal levels - where the energy release should be observable. Moreover, better knowledge of the flare energy distribution, of the relationship between energy release events in different wavelength bands, and of the loop length distribution and aspect ratio, is badly needed. Future solar space missions, like LEST and SOHO, may lead to a substantial improvement in this respect.

Among the problems to be addressed theoretically, we may mention also that the threshold behavior required in the nanoflare energy release process is still to 

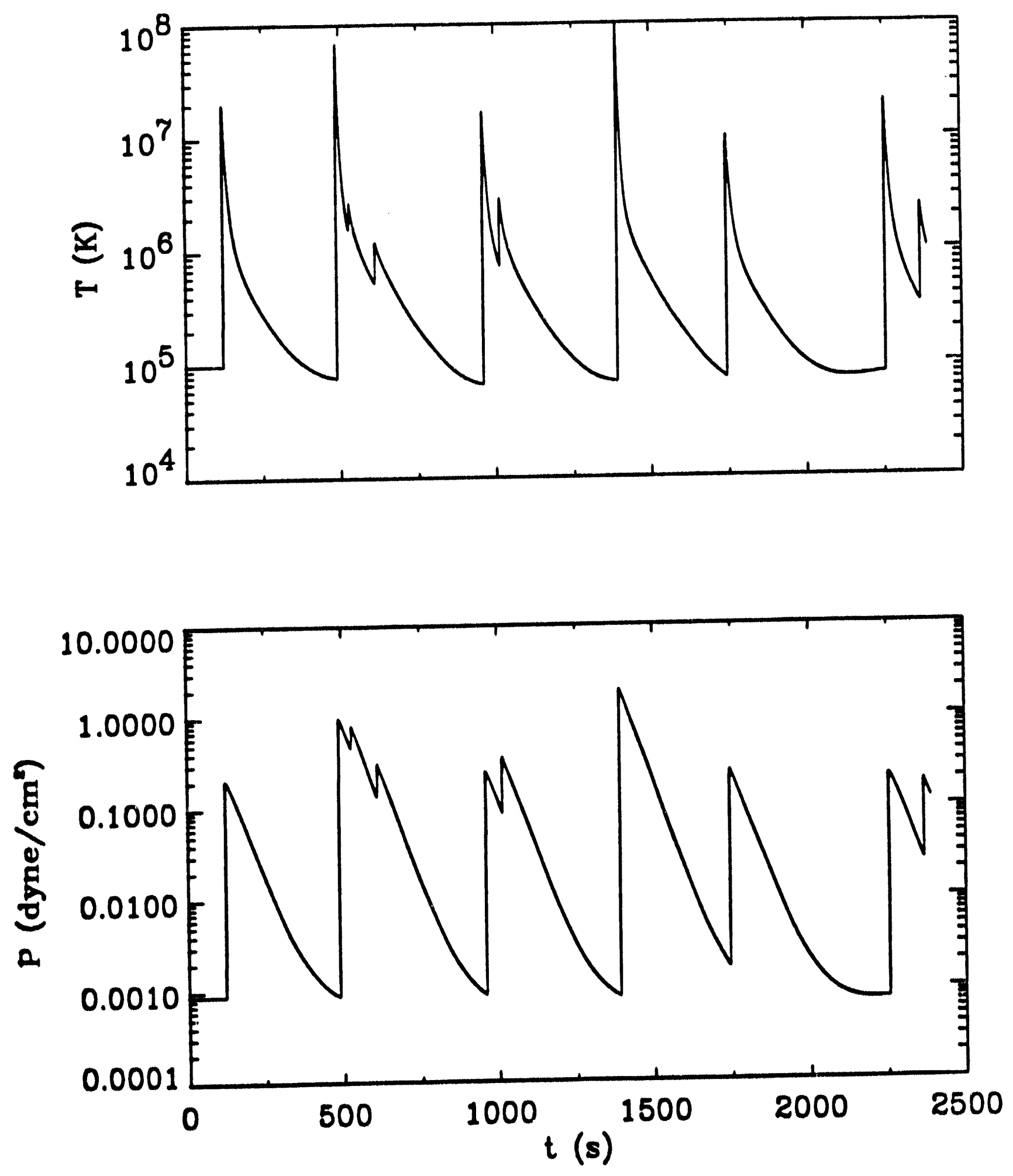

Fig. 1. Time profiles of temperature and pressure for an initially cool loop with $\mathrm{L}=2000 \mathrm{~km}$. The simulation extends over a time interval of $2388 \mathrm{~s}$, during which 10 nanoflares occur at random times and with a random (power-law) distribution of energies $(\alpha=2.2)$. 
be demonstrated and the value of the threshold has still to be established. The credibility of even a simple model like the one presented here will gain substantially from observational and theoretical developments. It is also worth pointing out that simulations analogous to these are still entirely lacking for stellar coronae: although subject to even greater uncertainties with respect to the solar case, such calculations would add to our overall confidence in the basic mechanisin. A further topic that needs investigation is the possibility of interpreting large flares as the cumulative effect of a number of nearly simultaneous nanoflares (de Jager and de Jonge 1978, Sturrock et al. 1984, Lu and Hamilton 1991). Nanoflares have opened a research area full of exciting perspectives. It remains for us to explore them fully.

\section{References}

C.J. Butler, M. Rodonò, B.H. Foing, B.M. Haisch (1986): Nature 321, 679.

C.-C. Cheng, R. Pallavicini (1991): Astrophys. J. 381, 234.

C. de Jager, G. de Jonge (1978): Solar Phys. 58, 127.

G.H. Fisher, R.C. Canfield, A.N. McClymont (1985): Astrophys. J. 289, 414.

W.M. Glencross (1975): Astrophys. J. 199, 1.53.

T. Gold (1964): "The Physics of Solar Flares", ed. by W. Hess, NASA.SP 50, 389.

H.S. Hudson (1991): Solar Phys. 133, 357.

R.A. Kopp, G. Poletto (1993): Astrophys. J., in press.

R.H. Levine (1974): Astropys. J. 190, 457.

E.T. Lu, R.J. Hamilton (1991): Astrophys. J. 380, 189.

P. MacNeice (1986): Solar Phys. 103, 47.

J.T. Mariska (1987): Astrophys. J. 319, 465.

R. Pallavicini, G. Serio, S. Vaiana, L. Acton, J. Leibacher, R. Rosner (1983): Astrophys. J. 270, 270.

E.N. Parker (1983): Astrophys. J. 264, 6.42.

E.N. Parker (1988): Astrophys. J. 330, 474.

E.N. Parker (1991): "Reviews in Modern Astronomy" 4, ed. by (i. Klare, 1.

J.G. Porter, J. Toomre, K.B. Gebbie (1984): Astrophys. J. 283, 879.

J.G. Porter, J.M. Fontenla, G.M. Simnett (1992): Astrophys. J., submitted.

F. Reale, G. Peres, S. Serio, R. Rosner, J.H.M.M. Schmitt (1988): Astrophys. J. 328, 256.

J.F. Vesecky, S.K. Antiochos, J.H. Underwood (1979): Astrophys. J. 233, 1979.

G.L. Withbroe, R.W. Noyes (1977): Ann. Rev. Astron. Astrophys, 15, 363. 

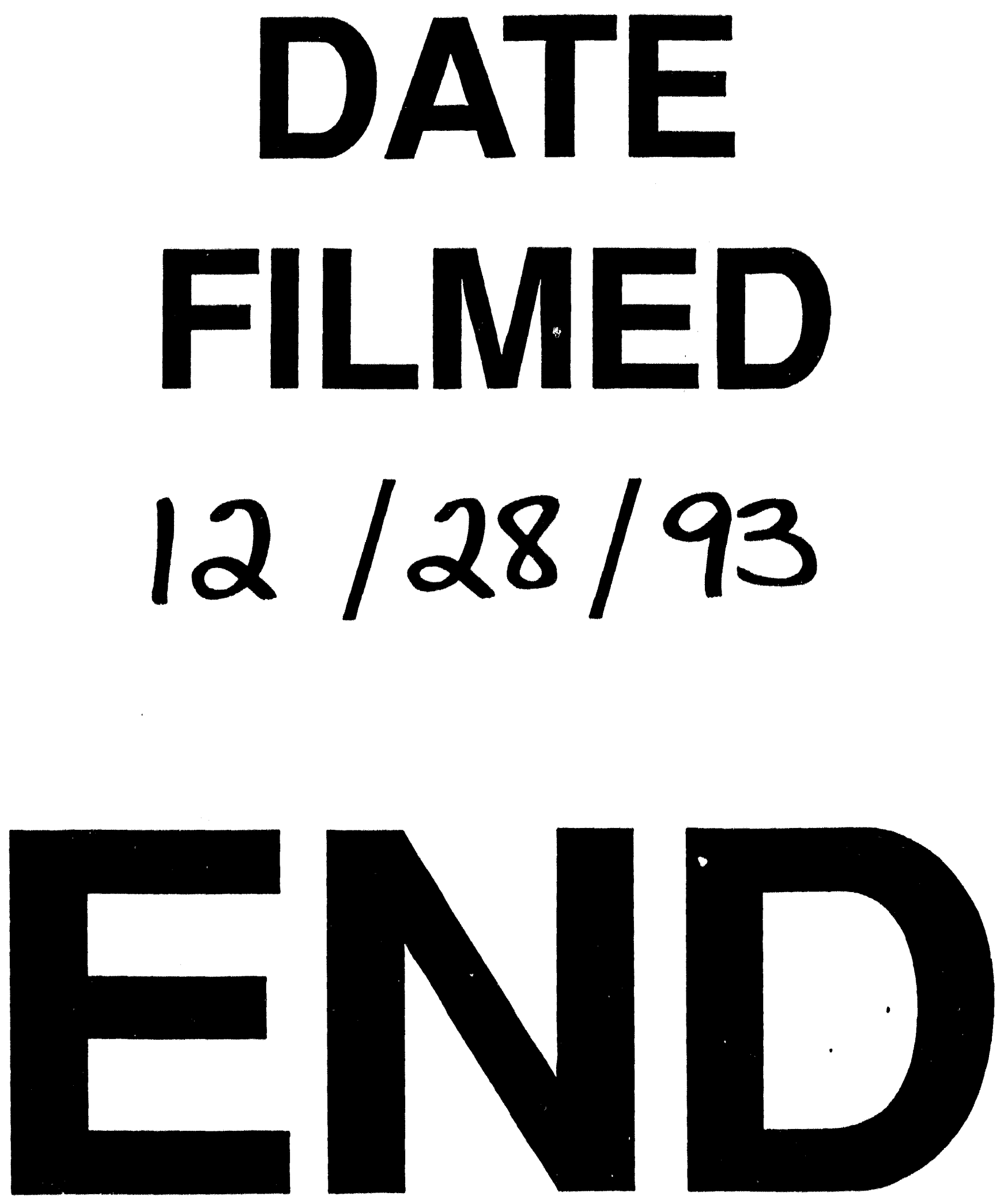


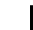

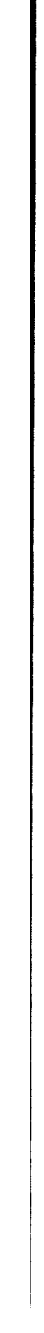

